\title{
Characterization of Porcine Endogenous Retrovirus Clones from the NIH Miniature Pig BAC Library
}

\author{
Seong-Lan Yu, ${ }^{1}$ Woo-Young Jung, ${ }^{1}$ Kie-Chul Jung, ${ }^{1,2}$ In-Cheol Cho, ${ }^{3}$ Hyun-Tae Lim, ${ }^{4}$ \\ Dong-Il Jin, ${ }^{1}$ and Jun-Heon Lee ${ }^{1}$ \\ ${ }^{1}$ Department of Animal Science \& Biotechnology, College of Agriculture and Life Sciences, Chungnam National University, \\ Daejeon 305-764, Republic of Korea \\ ${ }^{2}$ Research and Analysis Division, National Agricultural Products Quality Management Service, Gyungki 430-824, Republic of Korea \\ ${ }^{3}$ Subtropical Animal Experiment Station, National Institute of Animal Science, Jeju 690-150, Republic of Korea \\ ${ }^{4}$ Division of Applied Life Science, Gyeongsang National University, Jinju 660-701, Republic of Korea
}

Correspondence should be addressed to Jun-Heon Lee, junheon@cnu.ac.kr

Received 27 April 2011; Revised 8 June 2011; Accepted 16 June 2011

Academic Editor: Guihua H. Bai

Copyright (c) 2012 Seong-Lan Yu et al. This is an open access article distributed under the Creative Commons Attribution License, which permits unrestricted use, distribution, and reproduction in any medium, provided the original work is properly cited.

\begin{abstract}
Pigs have been considered as donors for xenotransplantation in the replacement of human organs and tissues. However, porcine endogenous retroviruses (PERVs) might transmit new infectious disease to humans during xenotransplantation. To investigate PERV integration sites, 45 PERV-positive BAC clones, including 12 PERV-A, 16 PERV-B, and 17 PERV-C clones, were identified from the NIH miniature pig BAC library. The analysis of 12 selected full-length sequences of PERVs, including the long terminal repeat (LTR) region, identified the expected of open reading frame length, an indicative of active PERV, in all five PERV-C clones and one of the four PERV-B clones. Premature stop codons were observed in only three PERV-A clones. Also, eleven PERV integration sites were mapped using a 5000-rad IMpRH panel. The map locations of PERV-C clones have not been reported before, thus they are novel PERV clones identified in this study. The results could provide basic information for the elimination of site-specific PERVs in selection of pigs for xenotransplantation.
\end{abstract}

\section{Introduction}

Xenotransplantation is the transplantation of living cells, tissues, and organs from one species to another. Xenotransplantation can be beneficial in overcoming the shortage of human organs and tissues for allotransplantation. Pigs are considered as the best xenotransplant organ donor due to anatomical and physiological similarities with humans, and the relatively less financial and ethical problems compared with primates. However, the use of porcine samples for human xenotransplantation suffers from possible infectious risks due to the transmission of porcine endogenous retroviruses (PERVs) from pigs to humans [1,2]. A recent study reported that PERVs can be transmitted to human cells via supernatants of primary porcine liver cells for short term using a bioartificial liver (BAL) model [3]. Despite this report, more than 200 human patients who received pig cells or tissues did not display PERV transmission in the blood cells [4]. Another study reported the absence of PERV transmission from 21 patients receiving porcine islets and Sertoli cells for 4.6-8 years [5]. Conversely, another study suggested that PERVs and human endogenous retroviruses (HERVs) could possibly form new viruses by recombination [4], complicating the transmission of PERV infection via xenotransplantation.

PERVs are present in the genome of all pig breeds and are classified as three main types: PERV-A, PERV-B and PERV-C. PERV-A and PERV-B can infect human cells as polytropic viruses and PERV-C infects only porcine cells as an ecotropic virus [2]. A very recent study suggested that recombinant PERV-A and PERV-C might be important infectious risk factor in human cells [6]. Therefore, eliminating infectious PERVs is a crucial issue in xenotransplantation. For knockout of active PERVs in the porcine genome, accurate PERV integration sites have to be identified. However, PERV copy numbers differ among pig breeds $[7,8]$, and there are 
variations in PERV integration sites among breeds [9, 10]. Recent success in the knockdown of PERV expressions has been reported using small interfering RNA (siRNA) $[11,12]$. For these biomedical experiments, miniature pigs are widely used mainly because they have the similar organ sizes to human $[13,14]$.

Long terminal repeats (LTRs) in the virus are mainly composed of the U3, R, and U5 regions. LTRs have important roles for the integration, replication, and regulation of retrovirus expression. Sequences in the R and U5 regions can repress PERV transcription [15]. Moreover, the level of PERV expression is associated with copy number of a 39 bp repeat in the PERV U3 region, which has an LTR transcription factor binding site [16]. A recent analysis of PERV LTR structures using sequences derived from public database of pig genome identified structural differences in the U3 region [17].

To characterize PERVs in National Institutes of Health (NIH) miniature pigs, the NIH miniature pig Bacterial Artificial Chromosome (BAC) library was screened and BAC end-sequences were used for the construction of contig maps for PERV-positive BAC clones and investigation of PERV map locations in the pig genome. Also, full-length PERV sequences, along with LTR sequences, were presently obtained. These results should provide basic information for selection of pigs for xenotransplantation research.

\section{Materials and Methods}

2.1. BAC Library Screening. A NIH miniature pig BAC library of approximately 15,000 clones was screened for PERV-positive clones by PCR analysis using three envelopspecific primers. PERV-specific primers were used for the identification of the PERV-A, PERV-B, and PERV-C BAC clones (Table 1). Polymerase chain reaction (PCR) was performed for PERV type identification with $20 \mathrm{ng}$ template DNA, 1X PCR buffer, $2 \mathrm{mM} \mathrm{MgCl}_{2}, 10 \mathrm{mM}$ of each dNTP, $0.2 \mu \mathrm{M}$ of each primer and one unit of Taq polymerase (GeNet Bio, Republic of Korea). The thermal profiles included an initiation denaturation at $94^{\circ} \mathrm{C}$ for $5 \mathrm{~min}$, following 30 cycles of denaturation at $94^{\circ} \mathrm{C}$ for $30 \mathrm{sec}$, annealing at $60^{\circ} \mathrm{C}$ for env- $\mathrm{A}$ and $-\mathrm{B}$ and $56^{\circ} \mathrm{C}$ for env-C for $30 \mathrm{sec}$ and extension at $72^{\circ} \mathrm{C}$ for $30 \mathrm{sec}$ and then a final extension step at $72^{\circ} \mathrm{C}$ for $5 \mathrm{~min}$ using a PTC-200 Programmable Thermal Controller (MJ Research, USA). PCR products were analyzed on $2 \%$ standard Tris/Acetate/EDTA (TAE) agarose gels stained with ethidium bromide (EtBr).

2.2. BAC End-Sequencing and Construction of Contig Maps. The $5^{\prime}$ and $3^{\prime}$ BAC end-sequences (BESs) were obtained from 45 PERV-positive BAC clones using T7 and SP6 universal primers for the cycle sequencing reaction. PCR products were run on a model 3730XL automated DNA sequencer (Applied Biosystems, USA). After masking of the repetitive sequences in the BESs using RepeatMasker software (http://www.repeatmasker.org/), the sequences were subjected to BLAST searches against NCBI database (http://www.ncbi.nlm.nih.gov/BLAST). BAC contig maps were constructed using the primers designed from BESs with the PRIMER 3 software (http://frodo.wi.mit.edu/primer3/) (Table 2). PCR mixtures contained 20 ng of template DNA, 1X PCR buffer, $2 \mathrm{mM} \mathrm{MgCl}_{2}, 10 \mathrm{mM}$ of each dNTP, $0.2 \mu \mathrm{M}$ of each primer, and one unit of Taq polymerase (GeNet Bio, Republic of Korea). The PCR amplification was carried out in a PTC-200 Programmable Thermal Controller (MJ Research, USA) for 25 cycles of denaturation at $94^{\circ} \mathrm{C}$ for $30 \mathrm{sec}$, annealing at $56^{\circ} \mathrm{C}$ for $30 \mathrm{sec}$ and extension at $72^{\circ} \mathrm{C}$ for $30 \mathrm{sec}$ and then a final extension step at $72^{\circ} \mathrm{C}$ for $5 \mathrm{~min}$. PCR products were separated using the $2 \%$ standard TAE agarose gels stained with EtBr.

2.3. Deduced PERV Amino Acid Sequences and Analysis of LTR Region. The identified positive BAC clones were validated by sequencing to determine whether the BAC clones possessed full length of PERV including the gag, pol, and env genes. To ascertain whether PERV sequences could make correct virus proteins, the amino acid sequences of each gene were deduced from nucleotide sequences using the open reading frame finder (ORFfinder) at NCBI (http://www.ncbi.nlm.nih.gov/gorf/gorf.html). To characterize the LTR of each clone, nucleotide sequences of the LTR were aligned using ClustalW program (http://www.ebi.ac.uk/ Tools/msa/clustalw2/) [21]. Repeat sequences in the LTR region were investigated using a tandem repeat finder [22].

2.4. PERV Mapping with IMpRH Panel. Chromosomal locations of PERV-positive clones were identified using the INRA/University Minnesota porcine Radiation Hybrid (IMpRH) panel $[23,24]$ using primers originating from BESs in the PERV-positive BAC clones (Table 2). PCR conditions were initiation heating at $94^{\circ} \mathrm{C}$ for $30 \mathrm{sec} ; 35$ cycle of at $94^{\circ} \mathrm{C}$ for $30 \mathrm{sec}$, at optimal annealing temperature of each primer set for $30 \mathrm{sec}$, at $72^{\circ} \mathrm{C}$ for $30 \mathrm{sec}$; a final elongation at $72^{\circ} \mathrm{C}$ for 5 min using a PTC-200 Programmable Thermal Controller (MJ Research). The PCR mixture included $25 \mathrm{ng}$ templates, 1X PCR buffer, $2 \mathrm{mM} \mathrm{MgCl} 2,10 \mathrm{mM}$ of each dNTP, $0.2 \mu \mathrm{M}$ of each primer, and one unit of Taq polymerase (GeNet Bio). The PCR products were sequenced to confirm whether correct target PCR products were amplified. The chromosomal assignments were determined using the IMpRH web server (http://imprh.toulouse.inra.fr).

\section{Results and Discussion}

3.1. Identification of PERV-Positive BAC Clones and BAC Contig Maps. Three types of PERV-positive clones were identified by PCR using three envelope-specific primers. From the 15,000 BAC clones in the library, forty-five PERV positive BAC clones were identified including 12 PERV-A, 16 PERV-B, and 17 PERV-C clones. In contrast to previous results that PERV-A and $-\mathrm{B}$ exist in the genomes of all pig breeds [25], the present study confirmed that the PERV$\mathrm{C}$ could be found in only a few pig breeds [26], which is different from PERV-A and PERV-B that were reported in all multitransgenic pigs generated for xenotransplantation. Another study documented high PERV-C copy numbers in 
TABLe 1: Type-specific primers for screening PERV-positive clones from NIH miniature pig BAC library.

\begin{tabular}{llcc}
\hline Gene & Primer sequence $\left(5^{\prime} \rightarrow 3^{\prime}\right)$ & Annealing temperature $\left({ }^{\circ} \mathrm{C}\right)$ & PCR product $(\mathrm{bp})$ \\
\hline \multirow{2}{*}{ Env-A } & F: TCCGTGCTTACGGGTTTAC & 60 & 224 \\
\hline \multirow{2}{*}{ Rnv-B } & F: TAGCCAATCTTTCCATCTCC & 60 & 192 \\
\hline \multirow{2}{*}{ Env-C } & R: CCGGAATCACACCTCCCAACC & 56 & \multirow{2}{*}{310} \\
\hline
\end{tabular}

the genome of the miniature pig line [25]. The present study also identified a relatively high percentage of PERV-C clones (17/45; 38\%) among 45 PERV-positive BAC clones.

To construct BAC contig maps, $5^{\prime}$ and $3^{\prime}$ BAC ends from 45 PERV-positive BAC clones were sequenced and primers for contig mapping were designed from the $90 \mathrm{BESs}$ derived from both T7 and SP6 ends (Table 2). Of these, BLAST searches against the NCBI database indicated that nine clones (2 PERV-A, 1 PERV-B and 6 PERV-C clones) were already present in the GenBank database (http://www .ncbi.nlm.nih.gov/genbank/). Before designing primers for the contig mapping, repetitive sequences were screened using RepeatMasker software (http://repeatmasker.org). Fifty-two BESs derived from 26 T7 primer ends and 26 SP6 primer ends were successfully used to construct contig maps of PERV-positive BAC clones. Sixteen PERV BAC clones (seven PERV-A clones (A1-1F, A1-1E, A1-2G, A4-1H, A1-6E, A16C, and A4-1G); four PERV-B clones (B3-7F, B4-13H, B18D, and B3-7G); five PERV-C clones (C1-1D, C4-2G, C110G, C3-1E, and C3-4B)) were mapped using both T7 and SP6 end-sequences. However, 10 PERV-positive BAC clones (1 PERV-A clone (A4-14H); 5 PERV-B clones (B1-7A, B311C, B3-3G, B4-1B and B3-12H); 4 PERV-C clones (C3$6 \mathrm{~F}, \mathrm{C} 2-5 \mathrm{~F}, \mathrm{C} 1-9 \mathrm{~B}$, and $\mathrm{C} 1-9 \mathrm{D})$ ) were only mapped with T7 ends. Also, 10 PERV-positive BAC clones (2 PERV-A clones (A3-9F and A3-10E); five PERV-B clones (B2-10D, B2-11A, B1-11C, B4-2E, and B3-12E); 3 PERV-C clones (C2$6 \mathrm{H}, \mathrm{C} 2-3 \mathrm{~F}$, and $\mathrm{C} 2-6 \mathrm{C})$ ) were only mapped with SP6 ends. Especially, one PERV-A clone (A4-1G), one PERV-B clone (B3-7G) and three PERV-C clones (C1-10G, C3-1E, and C348) did not overlap with other contigs and so were designated as singletons. On the other hand, nine PERV-positive BAC clones (two PERV-A clones (A3-7A and A3-5B); two PERV$\mathrm{B}$ clones (B1-11F and B1-11G); five PERV-C clones (C1-12A, C1-12C, C1-2D, C1-5F, and C3-5G)) could not be mapped with both T7 and SP6 ends. The results indicated that $42.2 \%$ (38/90) of the BESs contained repetitive sequences and could not be used to design primers for construction of contig maps due to the presence of large portion of repetitive sequences in the BESs (Figure 1).

3.2. PERV Amino Acid Sequences and Analysis of LTR Region. Because PERVs can be transmitted in a Mendelian fashion from parent to offspring through multiple generations, a large number of PERVs are known to be inactive. Therefore, deducing the amino acid sequence of a PERV is important to identify possibly active PERVs. Full-length PERV nucleotide sequences were obtained from 12 selected positive BAC clones containing three PERV-A (A1-1F, A1-6C, and A4$1 G$ ), four PERV-B (B3-7F, B3-3G, B3-7G, and B3-12E) and five PERV-C (C1-9B, C4-2G, C1-10G, C2-6C, and C3-1E). The nucleotide sequences were deposited in the GenBank database (Accession numbers HQ536005-HQ536016). All three selected PERV-A clones had low possibility to be active viruses because they had nonsense mutations in the envelope protein. However, one clone (B3-7F) from four PERV-B BAC clones and five PERV-C BAC clones had an intact open reading frame (ORF) and so a high possibility of the production of virus particles, indicating that they were active PERVs (Figure 2). Even though PERV-C alone could not infect human cells, the PERV-A and PERV-C recombinant types can increase infectious risk in relation to xenotransplantation [27]. Therefore, identification of pig lines free of PERV-C is also very important.

LTR sequences from 12 selected PERV-positive BAC clones were characterized. The identified sequence homologies in the LTR R and U5 regions were $96.5 \%-100 \%$. The possible reason for this high sequence conservation is that these two regions had TATA signal and cap sites related with transcriptional initiation. However, a previous study reported variable repeat numbers and differences in length of the U3 region [17]. Presently, similar results were obtained for the differences in repeat sequences in U3 regions (Figure 2). Three PERV-A LTRs were investigated and the sequence results revealed similar repeat patterns. Analyses of four PERV-B LTRs indicated that one (clone ID: B3$7 F)$ displayed differences in repeat numbers. One of the PERV-C LTRs (clone ID: C3-1E) had an insertion in the U3 region and another PERV-C LTR (clone ID: C1-10G) had a $98 \mathrm{bp}$ tandem repeat sequence in the U3 region. Recent studies associated the expression activity of PERV with the methylation status of LTRs $[28,29]$. Also, LTR elements might be used for the prediction of transcription activity as well as evolution because of insertions and deletions in the LTR region due to recombination event among different PERV types [17]. Two PERV-C clones (clone ID: C1-10G and C3-1E) had recombinant PERVs in the LTR region; further research should be carried out with these recombinant PERVs, especially when the pigs are used for the xenotransplantation research studies.

3.3. PERV Integration Sites. To investigate PERV map locations in the porcine genome, a linker-mediated PCR method was applied. Previously, PERV chromosomal integration sites were characterized in three pig breeds including Large White, 


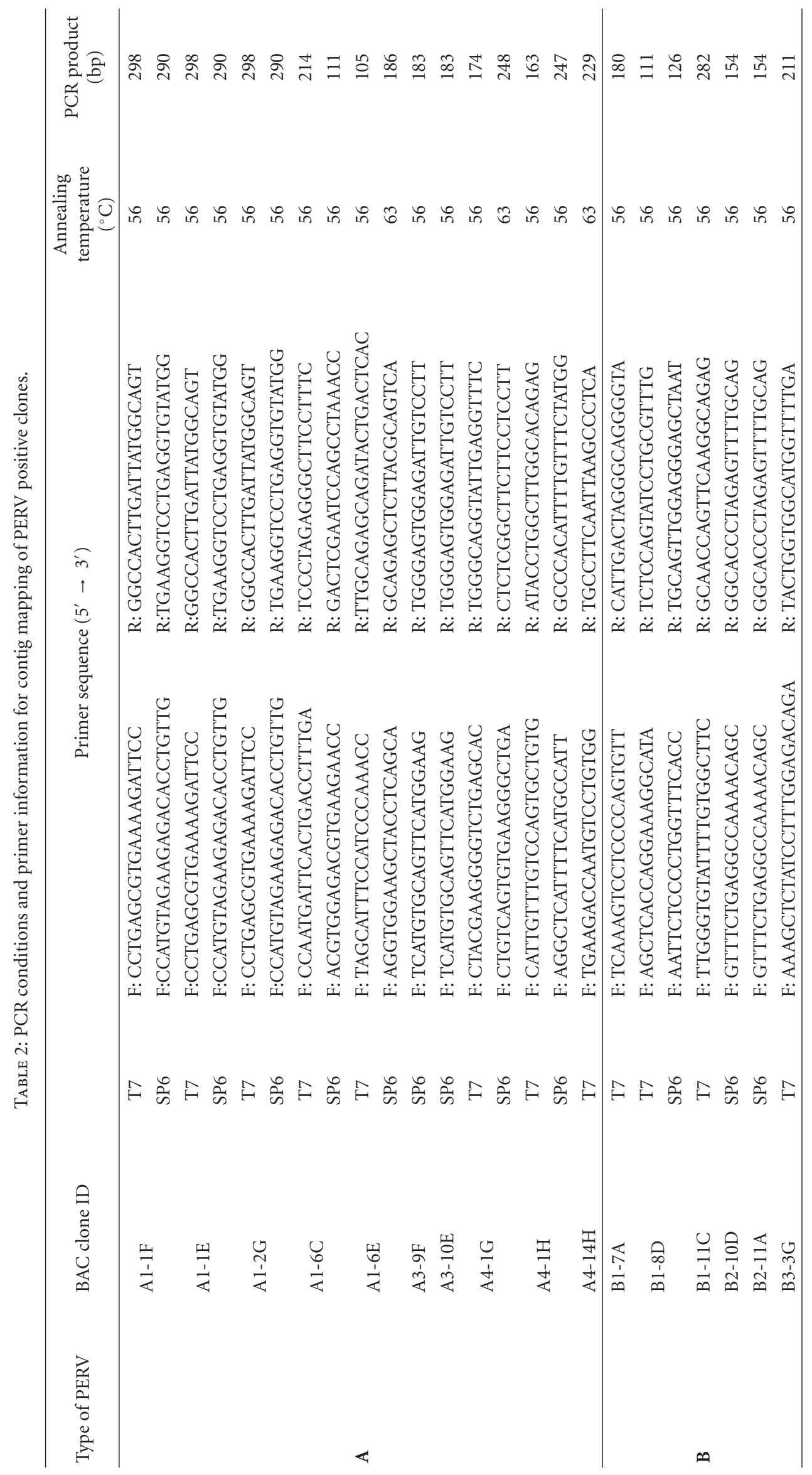




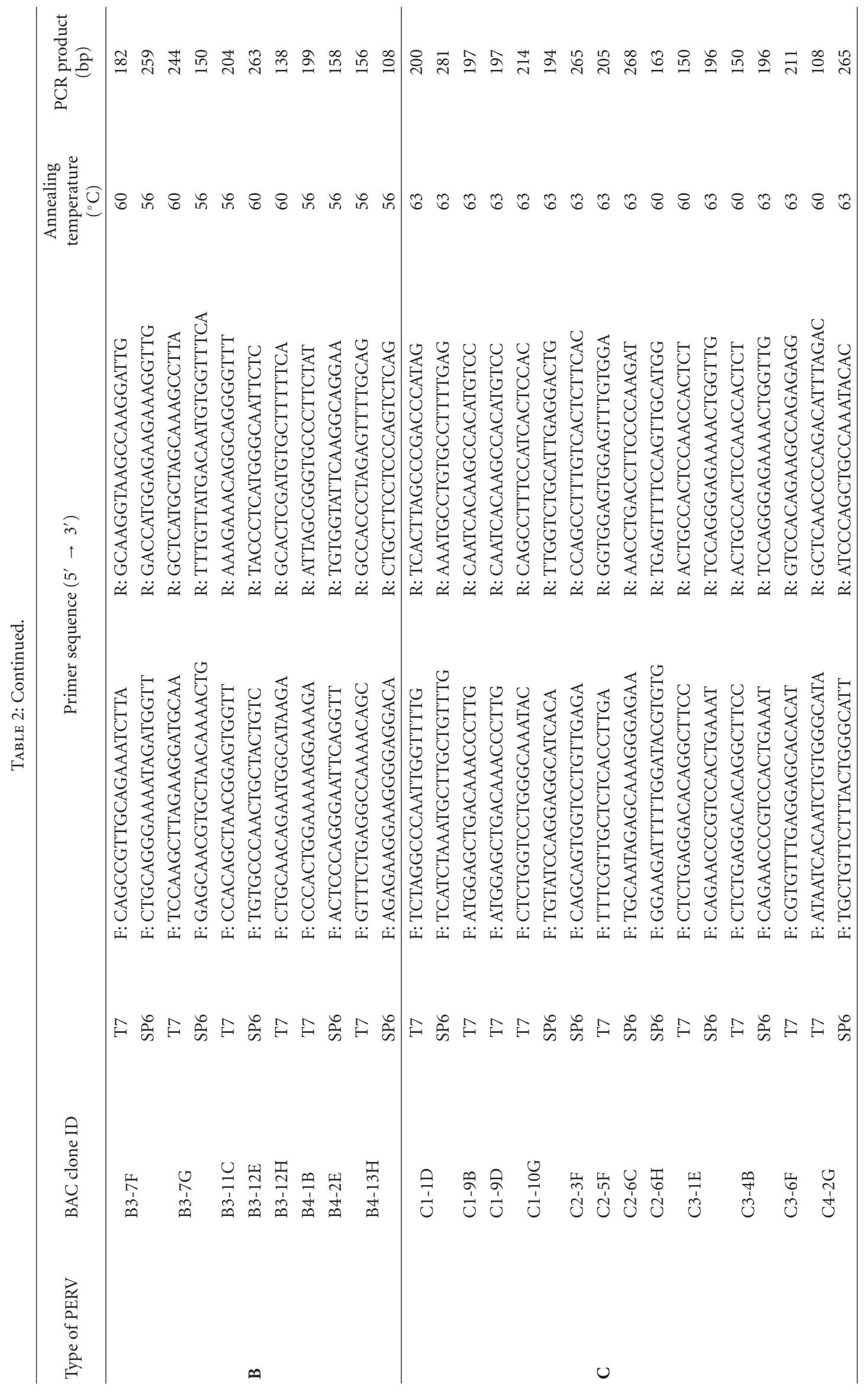




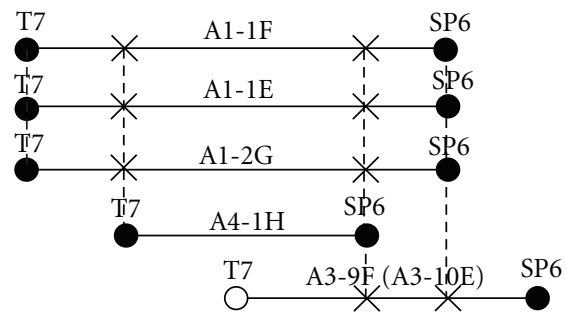

(a)

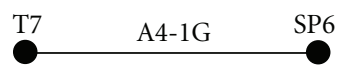

(c)

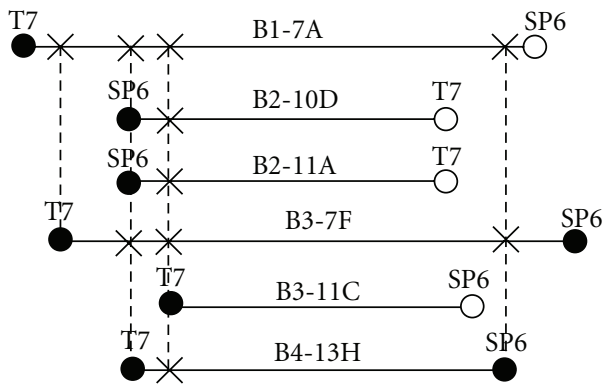

(e)

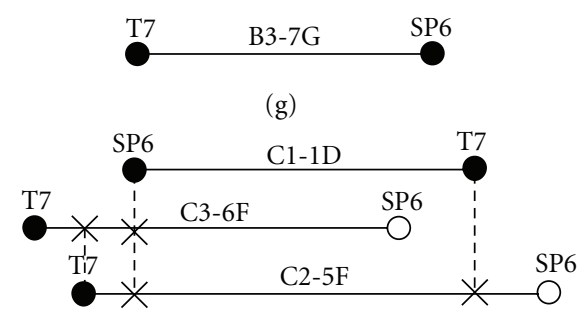

(i)

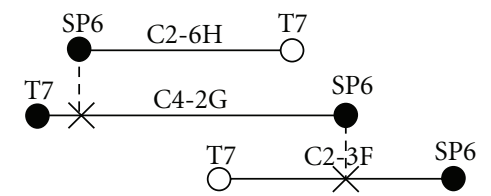

(k)

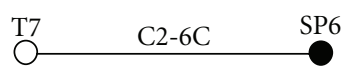

(m)

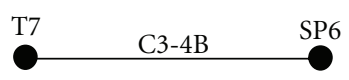

(o)

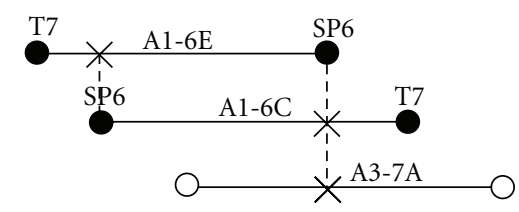

(b)

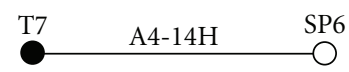

(d)

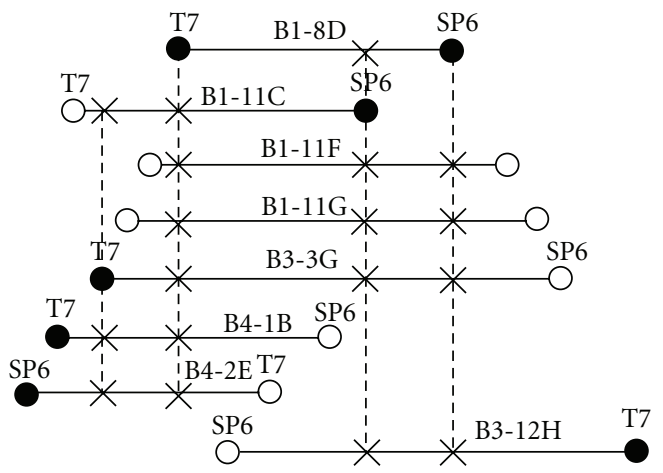

(f)

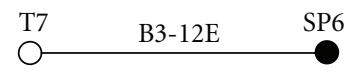

(h)

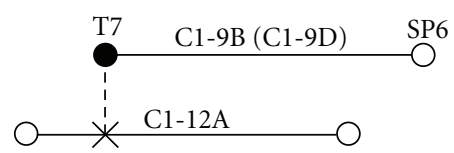

(j)

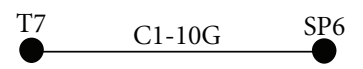

(l)

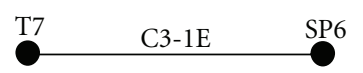

(n)

FIgURE 1: Contig maps for PERV-positive BAC clones using primers designed from BAC end-sequences. Fifteen PERV containing contig maps were constructed in this study ((a)-(d)) PERV-A type; ((e)-(h)) PERV-B type; ((i)-(o)) PERV-C type) and five contigs ((c) (g), (l), $(\mathrm{n})$ and $(\mathrm{o}))$ were identified as singletons. Solid circles indicate that primers were designed from the BAC end-sequences and open circles indicate that no primers were designed from the BAC end-sequences due to the repetitive sequences. Also, the presence of common $\mathrm{BAC}$ end-sequences among the PERV-positive BAC clones is indicated by cross mark. 


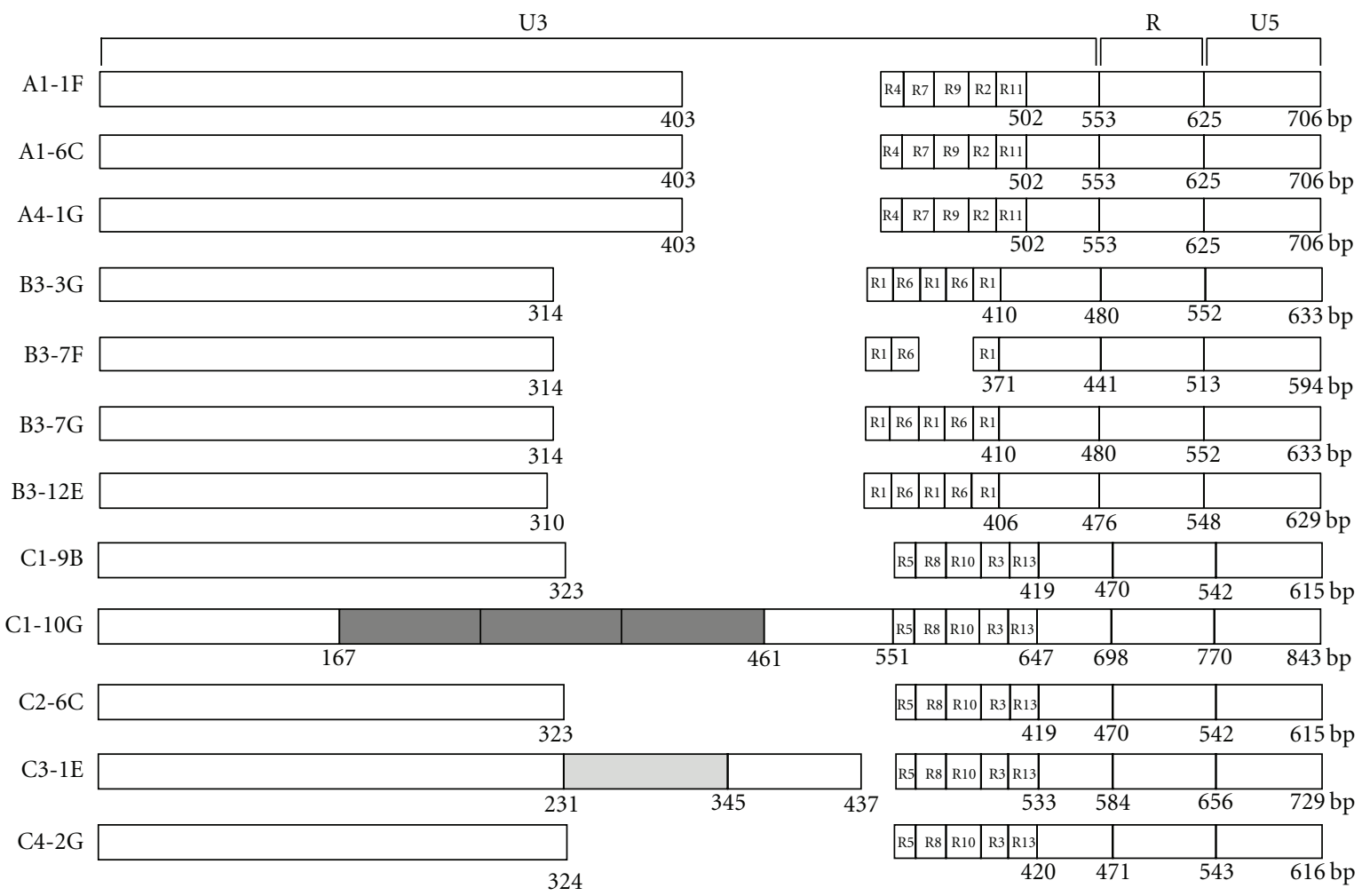

(a)

GCTCCTAACTGCT TGT T TGGCT TCTGTAAACCTGCT TGCATAAGATAAAAAGAGGAGAAGTCAATT TGCCTAACGGACC CCAGTAAGATCGGGCGTGCC

CATAAAGATGAAGAAAAGGGAGT T TCTAACTGCTTGT TTAGCT TCTGTAAAACTGCT TGCAAAAGATAGAAGGAGAGG AGGT TAAT T TCTAAAGCAACC TCAAAT TGGT TGCGC

R1:TATT T TAAAATGAT TGGT (original 18 bp repeat)

R2 :TAT TT TAAAAATGATTAGT(subtype of 18 bp repeat)

R3:TA T T T T GAAA T GA T T GGT(subtype of 18 bp repeat)

R4: T TAAAA T TAAT TGGT(subtype of 18 bp repeat)

R5 : T T AAA T TAA T TGGT(subtype of 18 bp repeat)

R6 :CCACGGAGCGCGGGC TC TCGA (original 21 bp repeat)

R7 :CCACAAAGCGCGGGCTCTCGA(subtype of 21 bp repeat)

R8 :CCACGAAGCGCGGGC TCTCGA (subtype of 21 bp repeat)

R9 :AGTTTTGAAT TGACTGGT T TGCGA (24 bp repeat)

R10:AGT T T TAAAT TGAC TGGT TTGTGA(subtype of 24 bp repeat)

R11: T TGTAAAAGCGCGGGC T T TG(20 bp repeat)

R12 : T T G TAAAGCGCGGGC T T TG(19bp repeat)

(b)

FIGURE 2: The organization and structure of PERV LTRs identified from the NIH miniature pig BAC clones (a) and the different subrepeat sequences identified in PERV LTRs (b). 
TABle 3: Comparison of characterized PERV-A, PERV-B and PERV-C integration sites in different four breeds.

\begin{tabular}{|c|c|c|c|c|c|c|c|c|c|}
\hline \multirow{2}{*}{$\begin{array}{l}\text { Pig } \\
\text { chromosome }\end{array}$} & \multicolumn{3}{|c|}{ NIH miniature pig } & \multicolumn{2}{|c|}{ Korean native pig ${ }^{1}$} & \multicolumn{2}{|c|}{ Large White pig $^{2}$} & \multicolumn{2}{|c|}{ Westran pig $^{3}$} \\
\hline & $\mathrm{A}$ & $\mathrm{B}$ & $\mathrm{C}$ & $\mathrm{A}$ & $\mathrm{B}$ & $\mathrm{A}$ & B & A & $\mathrm{B}$ \\
\hline 1 & & & & & & $\begin{array}{l}1 \mathrm{q} 2.1 \\
1 \mathrm{q} 2.3 \\
1 \mathrm{q} 2.4\end{array}$ & & $1 \mathrm{q} 1.2$ & $1 \mathrm{p} 2.2$ \\
\hline 2 & $2 \mathrm{q} 1.3-\mathrm{q} 2.1$ & & & & $\begin{array}{c}2 \mathrm{q} 2.1 \text { or } \\
2 \mathrm{q} 2.2\end{array}$ & & $2 \mathrm{q} 2.1$ & $2 \mathrm{p} 1.4$ & \\
\hline 3 & & $\begin{array}{l}3 \mathrm{q} 2.1-\mathrm{q} 2.7 \\
3 \mathrm{q} 1.1-\mathrm{q} 1.4\end{array}$ & & & & & & $3 \mathrm{p} 1.4$ & \\
\hline 4 & & 4 & & & & & $4 \mathrm{p} 1.1$ & & \\
\hline 5 & & & & & & & & $\begin{array}{c}5 \mathrm{p} 1.3 \\
5 \mathrm{p} \\
1.2 \\
5 \mathrm{q} \\
1.2 \\
5 \mathrm{q} 2.1\end{array}$ & $5 q 1.2$ \\
\hline 6 & $6 \mathrm{q} 3.5$ & & & & & & & $\begin{array}{l}6 \mathrm{p} 1.4 \\
6 \mathrm{q} 3.5\end{array}$ & \\
\hline 7 & & & & $7 \mathrm{p} 1.3$ & $7 \mathrm{p} 1.1$ & & $\begin{array}{c}\text { 7p1.1 } \\
7 \mathrm{p} 1.2-\mathrm{p} 1.1\end{array}$ & $\begin{array}{l}7 \mathrm{p} 1.3 \\
7 \mathrm{q} 1.5\end{array}$ & $7 \mathrm{p} 1.2$ \\
\hline 8 & & & & & & $8 \mathrm{p} 1.2$ & & & $8 \mathrm{p} 2.2$ \\
\hline 9 & & & & & $9 q 2.6$ & & $9 q 2.6$ & $9 q 2.4$ & $9 q 2.4$ \\
\hline 10 & & & & & & & $10 \mathrm{p} 1.2$ & & \\
\hline 11 & & & & & $\begin{array}{l}\text { 11q1.1 or } \\
11 \mathrm{q} 1.2\end{array}$ & & $\begin{array}{l}11 \mathrm{q} 1.2 \\
11 \mathrm{q} 1.4\end{array}$ & & $11 \mathrm{p} 1.3$ \\
\hline 12 & & & $12 \mathrm{p} 1.5$ & & & & & $12 \mathrm{p} 1.3$ & $12 \mathrm{q} 1.1$ \\
\hline 13 & 13 & & $\begin{array}{c}13 \\
13 q 4.1-q 4.9\end{array}$ & $\begin{array}{c}13 q 4.2 \text { or } \\
13 q 4.3\end{array}$ & & $\begin{array}{l}13 \mathrm{q} 4.2 \\
13 \mathrm{q} 4.3 \\
13 \mathrm{q} 4.9\end{array}$ & $13 \mathrm{q} 4.2$ & $13 \mathrm{q} 4.1$ & $13 \mathrm{q} 4.1$ \\
\hline 14 & & & & & & & $14 \mathrm{q} 2.8$ & & $14 \mathrm{q} 1.3$ \\
\hline 15 & & & 15 & & & & & & \\
\hline 16 & & & & & & & & 16q2.1 & $16 \mathrm{q} 2.1$ \\
\hline 17 & & & & $17 \mathrm{q} 1.2$ & $17 \mathrm{q} 2.1$ & & $17 \mathrm{q} 2.1$ & $17 \mathrm{q} 1.4$ & $17 \mathrm{q} 2.1$ \\
\hline 18 & & $18 \mathrm{q} 2.4$ & & & & & & & \\
\hline $\mathrm{X}$ & & & & & & & & Xp2.1 & Xp1.3 \\
\hline $\mathrm{Y}$ & & & & & & Yp1.2 & & Yp1.1 & $\mathrm{Yq}$ \\
\hline
\end{tabular}

${ }^{1}$ Published locations in Korean native pig [18].

${ }^{2}$ Published locations in Large White pig $[19,20]$.

${ }^{3}$ Published locations in Westran pig [10].

Westran, and Korean native pigs [10, 18-20]. In this research, 11 PERV genomic locations, consisting of three PERV-A, four PERV-B, and four PERV-C clones, were identified in $\mathrm{NIH}$ miniature pigs and compared with previously published integration sites in other three breeds. Eleven PERV-positive BAC clones were randomly selected from contig maps in each of the loci and the chromosomal locations have been determined using 5000-rad Radiation Hybrid panel [24] with primers designed from the BESs (Table 2). Of these, three map locations on SSC2, SSC6, and SSC13 were identified for the PERV-A clones. The PERV-positive BAC clone A4-14H, located on SSC6q3.5 and significantly linked with SW2419 marker (LOD score $=14.72$ ), corresponded to a site previously reported in the Westran pig. The fluorescence in situ hybridization (FISH) mapping technique used for the
PERV mapping in Westran pigs indicated that they are more possibly the same PERVs. Also, the PERV BAC clone A1-F1, located on SSC2q1.3-q2.1 and importantly linked with the SWR1342 marker (LOD score $=19.36$ ), represented a unique integration site not found in other three breeds. Also, the PERV BAC clone A1-6C mapped to SSC13 linked with the SW955 marker (LOD score $=4.18)$. Four PERV-B clones were mapped on SSC3, 4 and 18. Two PERV-positive BAC clones, B3-7G located on SSC3q1.1-q1.4 and B3-12E located on SSC3q2.1-q2.7, were highly linked with the SW1045 (LOD score $=8.66)$ and SW717 (LOD score $=18.47$ ) markers, respectively. Also, the PERV-positive BAC clone B3-7F was mapped on SSC 18q2.6 linked with the S0062 marker (LOD score $=5.64)$. Three PERV-B locations identified in NIH miniature pigs turned out to possess unique integration sites 
that have not been identified previously. Also, another PERV BAC clone, B3-3G, has been mapped on SSC4, which was linked to SW1003 marker (LOD score $=3.18$ ). According to previously reported integration sites, PERV-C types were not observed in Large White, Westran, and Korean native pigs. However, PERV-C clones were mapped in four chromosomal locations in the NIH miniature pigs. Two PERV-positive BAC clones, C1-10G located on SSC12p1.5 and C2-6C located on SSC13q4.1-q4.9, were significantly linked with the SW2490 $($ LOD score $=13.44)$ and SW769 $($ LOD score $=8.31)$ markers, respectively. The other two PERV positive BAC clones, $\mathrm{C} 3-6 \mathrm{~F}$ located on SSC15 and C4-2G located on SSC13, were linked with SW1892 $($ LOD score $=6.64)$ and S0084 $($ LOD score $=$ 7.81), respectively. These PERV-C-positive clones were not mapped previously; the present study is novel in identifying their map locations. In summary, only one PERV-A location on the SSC 6q3.5 (clone ID: A4-14H) in the NIH miniature pig correspond to integration site previously identified in the Westran pig, and six distinct retroviral integration sites were found on SSC 2, 3, 12, 13, and 18. On the remaining pig chromosomes, including $\mathrm{X}$ and $\mathrm{Y}$, any specific PERV genomic sites were not found in this study (Table 3 ).

In this study, 45 PERV-positive BAC clones were identified from the NIH miniature pig BAC library, indicating that the library is an important source for identifying PERV-containing clones. A previous report indicated that a miniature pig genome might contain a minimum of 30 genomic regions containing PERVs [8], therefore, 150 PERVcontaining clones should be found in the $\mathrm{NIH}$ miniature pig $\mathrm{BAC}$ library considering that the library has a $5 \mathrm{X}$ genome coverage. One of the reasons for this could be due to the complexity of PCR-based library screening techniques compared with the BAC filter-based methods, especially for screening the repetitive sequence as in PERVs. Along with this limitation, the genomic BAC library gave an important resource for identifying PERV-positive clones from NIH miniature pig. The full-length PERV nucleotide sequences identified in this study, together with the map information, can give important genomic information for the PERV researchers, especially for developing knockout strategy of the specific PERV. In conclusion, the PERV information in this study from the NIH miniature pig BAC library will provide valuable information for xenotransplantation studies, especially selection of pigs having specific PERV free animals. Also, this research can help the development of markers for screening the site-specific PERVs in the pig genome.

\section{Acknowledgments}

This work was supported by a Grant (no. 20070401034031) from BioGreen 21 program, Rural Development Administration, Republic of Korea and by a Grant (no. 20100023352) from the National Research Foundation of Korea, Republic of Korea. The authors gratefully acknowledge provision of pig radiation hybrid panel by Drs. Denis Milan and Martin Yerle (INRA, France).

\section{References}

[1] J. H. Blusch, C. Patience, and U. Martin, "Pig endogenous retroviruses and xenotransplantation," Xenotransplantation, vol. 9, no. 4, pp. 242-251, 2002.

[2] C. Patience, Y. Takeuchi, and R. A. Weiss, "Infection of human cells by an endogenous retrovirus of pigs," Nature Medicine, vol. 3, no. 3, pp. 282-286, 1997.

[3] J. H. Frühauf, H. Mertsching, S. Giri, N. R. Frühauf, and A. Bader, "Porcine endogenous retrovirus released bya bioartificial liver infects primary human cells," Liver International, vol. 29, no. 10, pp. 1553-1561, 2009.

[4] D. Louz, H. E. Bergmans, B. P. Loos, and R. C. Hoeben, "Reappraisal of biosafety risks posed by PERVs in xenotransplantation," Reviews in Medical Virology, vol. 18, no. 1, pp. 5365, 2008.

[5] R. Valdes-Gonzalez, L. M. Dorantes, E. Bracho-Blanchet, A. Rodríguez-Ventura, and D. J. G. White, "No evidence of porcine endogenous retrovirus in patients with type 1 diabetes after long-term porcine islet xenotransplantation," Journal of Medical Virology, vol. 82, no. 2, pp. 331-334, 2010.

[6] J. Denner, "Recombinant porcine endogenous retroviruses (PERV-A/C): a new risk for xenotransplantation?" Xenotransplantation, vol. 17, p. 120, 2010.

[7] D. E. Akiyoshi, M. Denaro, H. Zhu, J. L. Greenstein, P. Banerjee, and J. A. Fishman, "Identification of a full-length cDNA for an endogenous retrovirus of miniature swine," Journal of Virology, vol. 72, no. 5, pp. 4503-4507, 1998.

[8] P. Le Tissier, J. P. Stoye, Y. Takeuchi, C. Patience, and R. A. Weiss, "Two sets of human-tropic pig retrovirus," Nature, vol. 389, no. 6652, pp. 681-682, 1997.

[9] M. Gorbovitskaia, Z. Liu, N. Bourgeaux et al., "Characterization of two porcine endogenous retrovirus integration loci and variability in pigs," Immunogenetics, vol. 55, no. 4, pp. 262270, 2003.

[10] J. H. Lee, G. C. Webb, R. D. M. Allen, and C. Moran, "Characterizing and mapping porcine endogenous retroviruses in Westran pigs," Journal of Virology, vol. 76, no. 11, pp. 55485556, 2002.

[11] B. Dieckhoff, B. Petersen, W. A. Kues, R. Kurth, H. Niemann, and J. Denner, "Knockdown of porcine endogenous retrovirus (PERV) expression by PERV-specific shRNA in transgenic pigs," Xenotransplantation, vol. 15, no. 1, pp. 36-45, 2008.

[12] J. Ramsoondar, T. Vaught, S. Ball et al., "The piglet as a model for B cell and immune system development," Xenotransplantation, vol. 16, no. 3, pp. 164-180, 2009.

[13] J. S. Allan, G. A. Rose, J. K. Choo et al., "Morphometric analyses to predict appropriate donor size for swine-to-human cardiac xenotransplantation," Transplantation Proceedings, vol. 31, no. 1-2, pp. 975-977, 1999.

[14] C. A. Wilson, "Endogenous retroviruses: porcine endogenous retroviruses and xenotransplantation," Cellular and Molecular Life Sciences, vol. 65, no. 21, pp. 3399-3412, 2008.

[15] C. A. Wilson, S. Laeeq, A. Ritzhaupt, W. Colon-Moran, and F. K. Yoshimura, "Sequence analysis of porcine endogenous retrovirus long terminal repeats and identification of transcriptional regulatory regions," Journal of Virology, vol. 77, no. 1, pp. 142-149, 2003.

[16] G. Scheef, N. Fischer, E. Flory, I. Schmitt, and R. R. Tönjes, "Transcriptional regulation of porcine endogenous retroviruses released from porcine and infected human cells by heterotrimeric protein complex NF-Y and impact of immunosuppressive drugs," Journal of Virology, vol. 76, no. 24, pp. 12553-12563, 2002. 
[17] J. W. Huh, D. S. Kim, H. S. Ha et al., "Identification and molecular characterization of PERV Gammal long terminal repeats," Molecules and Cells, vol. 27, no. 1, pp. 119-123, 2009.

[18] W. Y. Jung, J. E. Kim, K. C. Jung et al., "Comparison of PERV genomic locations between Asian and European pigs," Animal Genetics, vol. 41, no. 1, pp. 89-92, 2010.

[19] C. Rogel-Gaillard, N. Bourgeaux, A. Billault, M. Vaiman, and P. Chardon, "Construction of a swine BAC library: application to the characterization and mapping of porcine type C endoviral elements," Cytogenetics and Cell Genetics, vol. 85, no. 3-4, pp. 205-211, 1999.

[20] C. Rogel-Gaillard, H. Hayes, N. Bourgeaux, and P. Chardon, "Assignment1 of two new loci for gamma 1 porcine endogenous retroviruses ( $\gamma 1$ PERV) to pig chromosome bands $2 \mathrm{q} 21$ and $11 \mathrm{q} 12$ by in situ hybridization," Cytogenetics and Cell Genetics, vol. 95, no. 1-2, pp. 112-113, 2001.

[21] M. A. Larkin, G. Blackshields, N. P. Brown et al., "Clustal W and Clustal X version 2.0," Bioinformatics, vol. 23, no. 21, pp. 2947-2948, 2007.

[22] G. Benson, "Tandem repeats finder: a program to analyze DNA sequences," Nucleic Acids Research, vol. 27, no. 2, pp. 573-580, 1999.

[23] D. Milan, R. Hawken, C. Cabau et al., "IMpRH server: an RH mapping server available on the web," Bioinformatics, vol. 16, no. 6, pp. 558-559, 2000.

[24] M. Yerle, P. Pinton, A. Robic et al., "Construction of a whole-genome radiation hybrid panel for high-resolution gene mapping in pigs," Cytogenetics and Cell Genetics, vol. 82, no. 3-4, pp. 182-188, 1998.

[25] T. Fujimura, S. Miyagawa, Y. Takahagi, T. Shigehisa, and H. Murakami, "Prevalence of porcine endogenous retroviruses in domestic, minature, and genetically modified pigs in Japan," Transplantation Proceedings, vol. 40, no. 2, pp. 594-595, 2008.

[26] B. Dieckhoff, B. Kessler, D. Jobst et al., "Distribution and expression of porcine endogenous retroviruses in multitransgenic pigs generated for xenotransplantation," Xenotransplantation, vol. 16, no. 2, pp. 64-73, 2009.

[27] J. C. Wood, G. Quinn, K. M. Suling et al., "Identification of exogenous forms of human-tropic porcine endogenous retrovirus in miniature swine," Journal of Virology, vol. 78, no. 5, pp. 2494-2501, 2004.

[28] K. C. Jung, D. M. Simond, C. Moran et al., "Investigation of deletion variation and methylation patterns in the $5^{\prime}$ LTR of porcine endogenous retroviruses," Asian-Australasian Journal of Animal Sciences, vol. 21, no. 11, pp. 1572-1584, 2008.

[29] S. J. Park, J. W. Huh, D. S. Kim et al., "Analysis of the molecular and regulatory properties of active porcine endogenous retrovirus gamma-1 long terminal repeats in kidney tissues of the NIH-Miniature pig," Molecules and Cells, vol. 30, no. 4, pp. 319-325, 2010. 

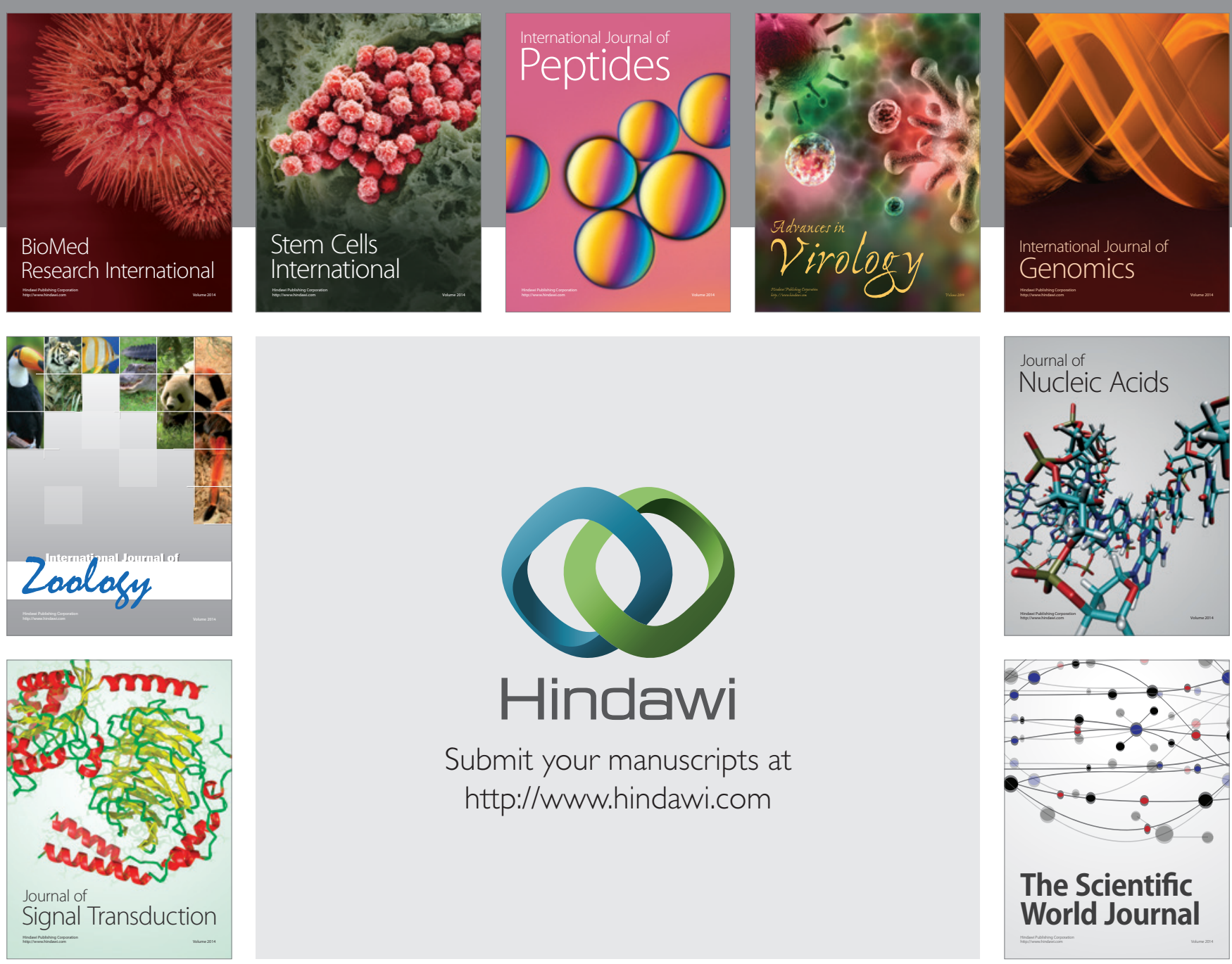

Submit your manuscripts at

http://www.hindawi.com
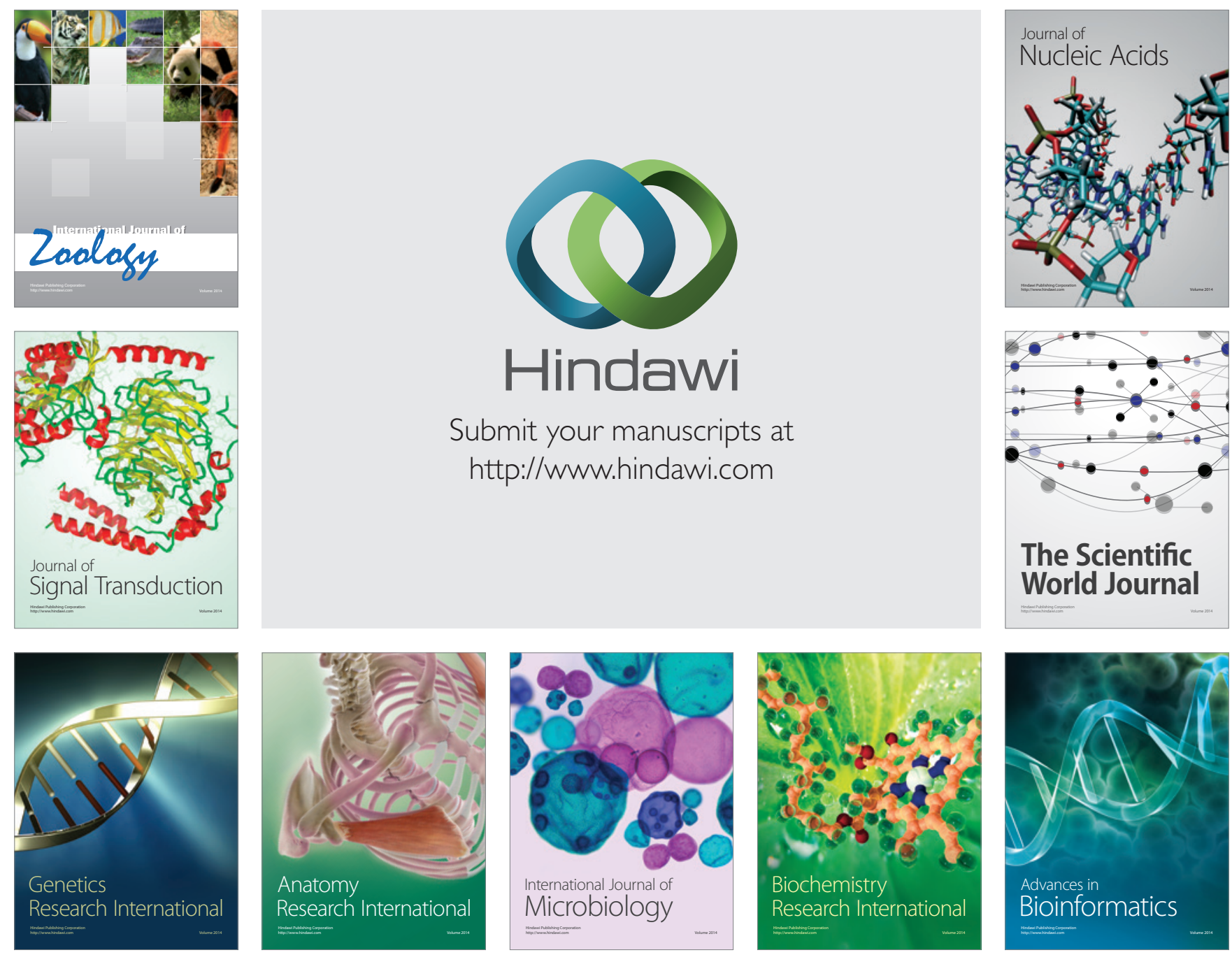

The Scientific World Journal
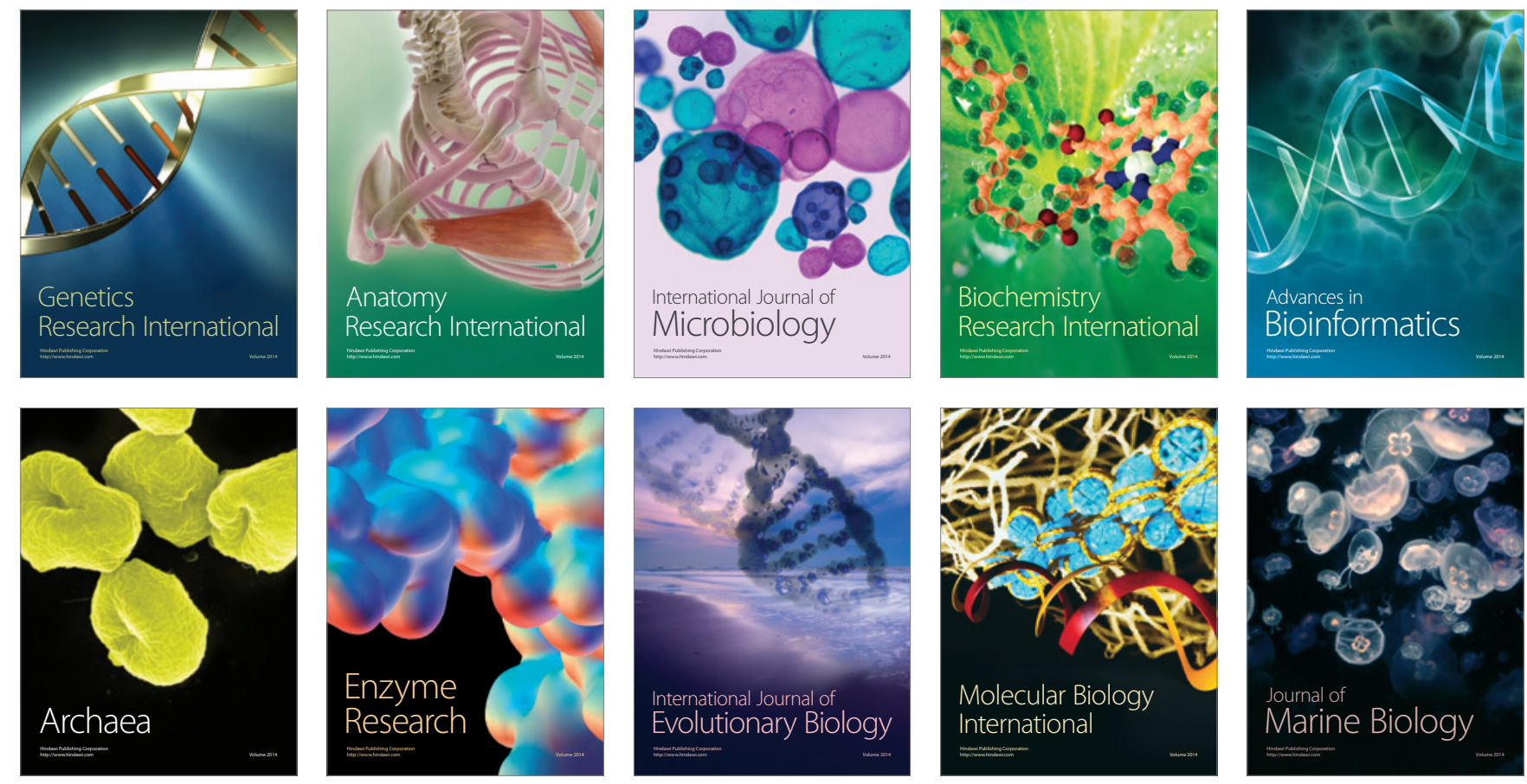